\title{
Vergleichung der Zusammensetzung des Caseins aus Frauen-, Kuh- und Ziegenmilch.
}

Von

Emil Abderhalden und Alfred Schittenhelm.

(Aus dem I. Chemischen Institut der Universität Berlin.)

(Der Redaktion zugegangen am 15. März 1906.)

Durch die «biologische Reaktion" ist erwiesen, daß das Casein der Milch verschiedener Tierarten offenbar ein verschiedenes ist. Die Beweisführung ist allerdings keine $\mathbf{z}$ wingende. Wir wissen vorläufig nicht, welche Produkte die Präcipitinbildung auslösen, und können uns wohl denken, daß die zu den Injektionen verwendeten Proteine Stoffe beigemengt enthielten, welche die Ursache der so außerordentlich empfindlichen, spezifischen Reaktionen sind. Von den verschiedenartigen Caseinen ist bis jetzt nur das der Kuhmilch ${ }^{1}$ ) eingehender untersucht worden. Sein Gehalt an Aminosäuren ist annähernd durch zahlreiche Abbauversuche festgestellt. Wir legten uns die Frage vor, ob die Caseine anderer Tierarten verschieden vom Kuhcasein sind, oder aber dieselben Bausteine besitzen und auch in denselben Mengenverhältnissen. Selbstverständlich können derartige Probleme bei Verbindungen so komplizierter Struktur nicht mit Hilfe von Elementaranalysen gelöst werden. Diese sagen gar nichts aus, und es können ohne Zweifel die heterogensten Eiweißsubstanzen genau dieselben Analysenwerte geben. Wir sind auch weit davon entfernt, behaupten zu wollen, da $B$ zwei Eiweißarten identisch sind, wenn sich nachweisen ließe, daß sie genau dieselben Aminosäuren in genau denselben Mengenver-

1) Emil Fischer, Hydrolyse des Caseins mit Salzsäure, Diese Zeitschrift, Bd. XXXIII, S. 151, 1901, und Emil Abderhalden, Aufbau und Abbau der Eiweißkörper im tierischen Organismus, Diese Zeitschrift, Bd. XLIV, S. 21, 1905. 
hältnissen enthalten. Selbstverständlich können die einzelnen Bausteine in ganz verschiedener Reihenfolge sich aneinanderreihen, ganz abgesehen von den isomeren Formen, die aus Gründen der Stereochemie denkbar sind. Von biologischen Gesichtspunkten aus hat der Nachweis, daß die Caseine verschiedener Milcharten gleiche Bausteine und in ähnlichen Mengenverhältnissen besitzen, wohl Interesse, denn wir wissen, daß im Darmkanal ein weitgehender Zerfall der Proteine stattfindet, und der Organismus sein Eiweiß von Grund aus neu aufbaut. Von diesem Gesichtspunkt aus kann ein ganz verschieden konstituiertes Protein doch biologisch gleichartig sein, sofern es nur bei der Aufspaltung die einzelnen Aminosäuren in gleichen Verhältnissen liefert und dem tierischen Organismus damit die Möglichkeit gibt, sein Körpereiweiß in derselben Weise ohne Verschleuderung von Material zu bilden. Auch hier müssen wir stets daran denken, daß das Gesetz des Minimums auch für den tierischen Organismus gilt, und daß die Eiweißsynthese sich unter Umständen nach dem im Minimum sich befindenden Baustein im Nahrungseiweiß richtet, wenn man nicht annehmen will, daß die tierische Zelle imstande ist, aus einer Aminosäure andere zu bilden. Die Möglichkeit einer derartigen Umwandlung ist nicht von der Hand zu weisen, dagegen muß betont werden, daß ein einwandfreier Beweis für derartige Prozesse noch aussteht.

Wir betonen die Schwierigkeit, oder klarer ausgedrückt die Unmöglichkeit, zwei Eiweißkörper nach unseren jetzigen Kenntnissen zu vergleichen oder gar für identisch in ihrem Aufbau zu erklären deshalb, weil trotz aller Fortschritte in der Eiweißchemie jetzt noch Behauptungen von der Umwandlung von einer Eiweißart in eine andere auf Grund einiger physikalischer Beobachtungen auftreten. ${ }^{1}$ )

Die folgende Tabelle gibt die erhaltenen Resultate wieder. Zum Vergleich sind die bei der Hydrolyse des Caseins aus Kuhmilch gewonnenen Werte mit angeführt.

1) Es sei z. B. verwiesen auf: Leopold Moll, Zur künstlichen Umwandlung von Albumin in Globulin, Hofmeister's Beiträge, Bd. VII, S. 311, 1905. 


\begin{tabular}{ll|c|c|c}
\hline & & \multicolumn{3}{|c}{ Casein aus } \\
\cline { 2 - 5 } & & Kuhmilch & Ziegenmilch & Frauenmilch \\
\hline Tyrosin . . . . . . . . . & 4,5 & 4,95 & 4,71 \\
Leucin . . . . . . . . . & 10,5 & 7,4 & - \\
Alanin . . . . . . . . . . & 0,9 & 1,5 & - \\
Prolin . . . . . . . . . & 3,1 & 4,62 & - \\
Phenylalanin . . . . . . . & 3,2 & 2,75 & - \\
Asparaginsäure . . . . . . & 1,2 & 1,1 & - \\
Glutaminsäure . . . . . . . & 10,7 & 11,25 & - \\
Diaminotrioxydodekansăure . & 0,75 & vorhanden & -
\end{tabular}

Ein Blick auf die Gegenüberstellung zeigt, daß zwischen der Zusammensetzung des Kuh- und Ziegenmilchcaseins eine weitgehende Ähnlichkeit besteht. Auch der Tyrosingehalt des Caseins aus Frauenmilch entspricht dem aus den beiden genanten Milcharten gewonnenen Casein.

\section{Experimenteller Teil.}

1. Casein aus Ziegenmilch. Dasselbe wurde nach der uiblichen Methode durch Fällung mit Essigsäure aus der 8-10fach mit Wasser verdünnten Milch und darauf folgendem Kolieren gewonnen. Die so erhaltene Substanz wurde noch zweimal mit Hilfe einiger Tropfen Natronlauge gelöst und mit Essigsäure wieder gefällt, endlich mit Wasser gewaschen und mit Alkohol und Äther extrahiert. Das Präparat enthielt nunmehr 9,7\% Wasser und 1,4\% Asche. Zur Hydrolyse wurden $220 \mathrm{~g}$ benutzt oder rund $200 \mathrm{~g}$ trockenen Materials. Dasselbe wurde mit 11 25\% iger Schwefelsäure 12 Stunden am Rückflußkühler gekocht, dann die Schwefelsäure quantitativ mit Baryt entfernt, und das Filtrat bis zur Kristallisation eingeengt. Der Baryumsulfatniederschlag wurde nun so lange mit Wasser ausgekocht, bis das Filtrat keine Reaktion mit Millon'schem Reagens mehr gab, und die Waschwasser mit dem ursprünglichen Filtrat von Baryumsulfat vereinigt eingeengt. Es wurden auf diese Weise 9,9 g Tyrosin erhalten. 
10,841 g Substanz gaben 0,4020 $\mathrm{g} \mathrm{CO}_{3}$ und $0,1015 \mathrm{~g} \mathrm{H}_{2} \mathrm{O}$

Berechnet für $\mathrm{C}_{8} \mathrm{H}_{\mathbf{1 1}} \mathrm{NO}_{8}$; Gefunden:

$59,66 \% \mathrm{C}$ und $6,07 \% \mathrm{H}$. $59,55 \% \mathrm{C}$ und $6,18 \% \mathrm{H}$.

Die Hauptmenge des Tyrosins wurde zum Nachweis etwa beigemengter Diaminotrioxydodekansäure in $500 \mathrm{ccm}$ ca. $5 \%$ iger Schwefelsäure gelöst und mit 5\% iger Phosphorwolframsäure gefällt. Der gesammelte und getrocknete Niederschlag wog 2,6 g. Er wurde in Wasser suspendiert, mit $5 \mathrm{~g}$ Baryt zusammen längere Zeit geschüttelt, und dann filtriert. Nach quantitativer Entfernung des Baryums durch Schwefelsäure wurde das Filtrat zur Trockene eingeengt. Es resultierte eine geringe Menge einer nach dem Umlösen in Wasser unter Anwendung von Tierkoble gegen $270^{\circ}$ unter teilweiser Zersetzung sublimierenden Substanz. Sie bildete ein in mikroskopisch kleinen Nädelchen kristallisierendes salzsaures Salz. Die Schwerlöslichkeit des Kupfersalzes spricht mit den erwähnten Eigenschaften dafür, daß höchst wahrscheinlich die von Emil Fischer und Emil Abderhalden ${ }^{1}$ ) im Casein aus Kuhmilch aufgefundene Diaminotrioxydodekansäure, $\mathrm{C}_{12} \mathrm{H}_{26} \mathrm{~N}_{2} \mathrm{O}_{5}$, vorlag.

Die vereinigten Filtrate von Tyrosin wurden unter vermindertem Druck bei $40^{\circ}$ des Wasserbades zur Trockene eingeengt, der Rückstand mit konzentrierter Salzsäure aufgenommen und bis zur Sättigung Salzsäuregas eingeleitet. Bald erfolgte Kristallisation. Sie war jedoch recht unvollständig und dunkel gefärbt. Wir lösten sie deshalb durch Zugabe von Wasser auf und kochten die Lösung unter Zugabe von Tierkohle, bis sie nunmehr hellgelb gefärbt war. Nun engten wir sie vorsichtig ein und sättigten sie annähernd mit Salzsäuregas. Es erstarrte bald die ganze Masse, die sich sehr leicht auf Koliertuch absaugen ließ. Sie wurde mit kalter konzentrierter Salzsäure und mit Alkohol gewaschen. Die über Kalk und Schwefelsäure getrocknete Masse wog 20,0 g. 2 g wurden durch Kochen mit Bleioxyd in die freie Säure übergeführt.

$0,1872 \mathrm{~g}$ Substanz gaben $0,2796 \mathrm{~g} \mathrm{CO}_{2}$ und $0,1042 \mathrm{~g} \mathrm{H}_{2} \mathrm{O}$

Berechnet für $\mathrm{C}_{8} \mathrm{H}_{8} \mathrm{NO}_{4}$ :

Gefunden :

$40,81 \% \mathrm{C}$ und $6,12 \% \mathrm{H}$. 40,73\% C und 6,19\% $\mathrm{H}$.

1) Emil Fischer u. Emil Abderhalden, Notizen über Hydrolyse von Proteinstoffen, Diese Zeitschrift, Bd. XLII, S. 540, 1904. 
Die Mutterlauge des Glutaminsäurechlorhydrates schied im Verlauf der nächsten Tage beim Stehen auf Eis noch $3,0 \mathrm{~g}$ des salzsauren Salzes ab. Sie wurde nach dessen Entfernung zum Sirup eingeengt und in der gewohnten Weise mit Alkohol und gasförmiger Salzsäure verestert. Die Ester wurden mit Alkali und Kaliumcarbonat in Freiheit gesetzt. Ihre fraktionierte Destillation ergab folgendes Resultat:

1. Fraktion bis $100^{\circ}$ (Temperatur des Wasserbades) bei $11 \mathrm{~mm}$ Druck $30,2 \mathrm{~g}$ 2. $>100^{\circ}(,>)>0,5>>15,0$ ? 3. $>200^{\circ}(>$ Ölbades $)>0,5>>17,0$,

Die Untersuchung der einzelnen Fraktionen geschah in der üblichen Weise.

\section{Fraktion.}

Sie wurde durch 8 stündiges Kochen mit der 7 fachen Menge Wasser am Rückflußkühler verseift und lieferte nach dem Eindampfen und Auskochen mit Alkohol eine Kristallisation von $11,6 \mathrm{~g}$, welche zum größten Teil aus Leucin bestand. Davon wurden $1,8 \mathrm{~g}$ Alanin abgetrennt.

Das isolierte Alanin hatte folgende Zusammensetzung:

$0,1747 \mathrm{~g}$ Substanz lieferten $0,2603 \mathrm{~g} \mathrm{CO}_{2}$ und $0,1251 \mathrm{~g} \mathrm{H}_{2} \mathrm{O}$

Berechnet für $\mathrm{C}_{8} \mathrm{H}_{7} \mathrm{NO}_{2}$ : Gefunden :

$40,45 \% \mathrm{G}$ und $7,87 \% \mathrm{H}$. $40,63 \% \mathrm{G}$ und $8,03 \% \mathrm{H}$.

\section{Fraktion.}

Sie wurde wie die erste verseift, fraktioniert und die einzelnen Fraktionen mit Alkohol ausgekocht. Danach betrug die letzte Fraktion 1,2 $\mathrm{g}$ und bestand aus Alanin. Die Hauptmenge $(6,0 \mathrm{~g})$ war Leucin (F. 2970 korr.).

Das isolierte Leucin hatte folgende Zusammensetzung:

$0,1679 \mathrm{~g}$ Substanz lieferten $0,3371 \mathrm{~g} \mathrm{CO}_{2}$ und $0,1494 \mathrm{~g} \mathrm{H}_{2} \mathrm{O}$

Berechnet für $\mathrm{C}_{8} \mathrm{H}_{18} \mathrm{NO}_{2}$ : Gefunden:

$54,92 \% \mathrm{C}$ und $9,99 \% \mathrm{H}$. $\quad 54,76 \% \mathrm{C}$ und $9,88 \% \mathrm{H}$.

Die aus Fraktion 1 und 2 erhaltenen vereinigten alkoholischen Auszüge wurden zur Gewinnung der Pyrrolidincarbonsäure im Vakuum zur Trockene verdampft und wiederholt mit abșolutem Alkohol aufgenommen. Der Rückstand der alkoholischen Auszüge gab mit Wasser und überschüssigem, frisch 
gefälltem Kupferoxyd gekocht, eine tiefblaue Lösung, welche nach dem Abfiltrieren des ungelösten Kupferoxyds bei $14 \mathrm{~mm}$ Druck zur Trockene verdampft wurde. Die Trennung des aktiven $(10,0 \mathrm{~g})$ vom racemischen $(2,6 \mathrm{~g})$ Kupfersalz wurde durch Auskochen des Rückstandes mit Alkohol bewirkt.

Das bei $120^{\circ}$ getrocknete racemische Kupfersalz zeigte folgende Zusammensetzung:

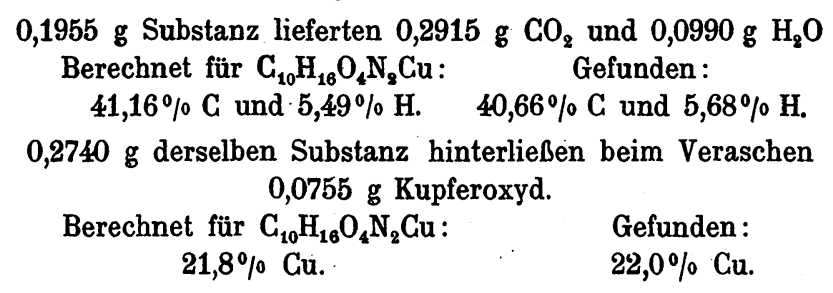

3. Fraktion.

Zur Darstellung des Phenylalanins wurde diese Fraktion mit der fünffachen Menge Wasser versetzt und mit Äther ausgeschüttelt. Der mit Wasser gut gewaschene Ätherauszug wurde durch Abdestillieren vom Äther befreit, und der im Rückstand befindliche Phenylalaninester durch Übergießen mit rauchender Salzsäure und Einengen in das Phenylalaninchlorhydrat übergeführt. Auf diese Weise wurden $6,4 \mathrm{~g}$ erhalten. Aus dem salzsauren Salz wurde in der gewohnten Weise durch Einengen mit Ammoniak die freie Säure gewonnen.

\section{0,1226 g Substanz gaben $0,2944 \mathrm{~g} \mathrm{CO}_{8}$ und $0,0736 \mathrm{~g} \mathrm{H}_{2} \mathrm{O}$ Berechnet für $\mathrm{C}_{9} \mathrm{H}_{11} \mathrm{NO}_{9}$ : $\quad$ Gefunden: $65,45 \%$ C und $6,66 \%$ H. $\quad 65,49 \%$ C und $6,67 \% \mathrm{H}$.}

Die nach dem Ausäthern zurückgebliebene wässerige Lösung der Ester wurde durch zweistündiges Kochen auf dem Wasserbad mit $20 \mathrm{~g}$ Baryt verseift und nach mehrtägigem Stehen die vom abgeschiedenen Baryt abfiltrierte Lösung mit Schwefelsäure quantitativ vom Baryt befreit. Der beim Eindampfen des Filtrates verbleibende Rückstand wurde mit rauchender Salzsäure angerührt und eingeengt. Daraus kristallisierten 5,0 g Glutaminsäurechlorhydrat aus.

Das Filtrat des Glutaminsäurechlorhydrats wurde durch Kochen mit Bleioxyd von der Mineralsäure befreit, und das ge- 
löste Blei aus dem Filtrat mit Schwefelwasserstoff entfernt. Aus der filtrierten Lösung gewannen wir 2,2 g Asparaginsäure. $0,2019 \mathrm{~g}$ Substanz gaben $0,2672 \mathrm{~g} \mathrm{CO}_{2}$ und $0,0973 \mathrm{~g} \mathrm{H}_{2} \mathrm{O}$

Berechnet für $\mathrm{C}_{4} \mathrm{H}_{7} \mathrm{NO}_{4}$ : Gefunden:

$36,09 \% \mathrm{C}$ and $5,26 \% \mathrm{H}$. $36,09 \% \mathrm{C}$ und $5,35 \% \mathrm{H}$.

Die Gesamtmenge der isolierten Monoaminosäuren betrug für $200 \mathrm{~g}$ Ziegencasein:

$\begin{array}{lr}\text { Alanin } & 3,0 \mathrm{~g} \\ \text { Leucin } & 14,8, \\ \text { Prolin } & \mathbf{9 , 2 4}, \\ \text { Phenylalanin } & \mathbf{5 , 5} \\ \text { Glutaminsäure } & \mathbf{2 2 , 5} \\ \text { Asparaginsäure } & \mathbf{2 , 2} \\ \text { Tyrosin } & \mathbf{9 , 9}\end{array}$

2. Casein aus Frauenmilch. Die Ausfällung des Caseins aus der sehr stark verdünnten Frauenmilch geschah durch Ansäuren mit Essigsäure und Einleiten von Kohlensäure, indem wir nach J. Schmidt') dabei eine Temperatur von $40^{\circ}$ innehielten. Da die Frauenmilch in großen Mengen schwer erhältlich ist, und noch dazu die Ausbeute an Casein sehr gering ist, so konnten wir uns nur etwas über $8 \mathrm{~g}$ darstellen. Das Präparat hatte einen Wassergehalt von $11,8 \%$, so daß wir also nur mit rund $7 \mathrm{~g}$ wasserfreier Substanz arbeiteten. Der Aschengehalt war $0,71 \%$. Das Casein wurde mit der 6 fachen Menge $25 \%$ iger Schwefelsäure am Rückflußkühler mehrere Stunden gekocht und danach wie beim Casein aus Ziegenmilch verfahren. Es konnten so $0,33 \mathrm{~g}$ Tyrosin isoliert werden.

Das Filtrat wurde auf wenige Kubikzentimeter eingeengt, diese mit gasförmiger Salzsäure gesättigt und weiter eingeengt. Auf diese Weise wurden $0,35 \mathrm{~g}$ Glutaminsäurechlorhydrat (=0,28 $\mathrm{g}$ freier Säure) isoliert.

3. Albumin aus Frauenmilch. Dasselbe wurde aus den Filtraten des Caseins durch Eindampfen gewonnen. Nach Behandlung mit Alkohol und Äther betrug die erhaltene Menge 18 g. Das Präparat hatte einen Wassergehalt von $9,61 \%$ und enthielt 3,42\% Asche. Auch dieses wurde wie das Casein aus

1) Thierfelder-Hoppe-Seyler, 1903, S. 543. 
Vergleichung der Zusammensetzung des Caseins usw. $\quad 465$

Frauenmilch hydrolysiert. Erhalten wurden $0,2 \mathrm{~g}$ Tyrosin und $0,2 \mathrm{~g}$ Glutaminsäurechlorhydrat $(=0,16 \mathrm{~g}$ freier Säure). Es entsprechen diese Mengen 1,26\% Tyrosin und 0,98\% Glutaminsäure, wenn man das wasserfreie Präparat in Rechnung zieht. Jedenfalls sind die Werte für die Glutaminsäure ganz erheblich zu niedrig. Wir hoffen nach dieser Richtung unsere Versuche ergänzen zu können. Jedenfalls ist der Tyrosingehalt des Caseins der Frauenmilch ein ähnlicher wie der der Kuhund Ziegenmilch. 